\section{Fragen an ...}

\section{Dr. Mike O. Karl, Dresden \\ Träger des EYEnovative Förderpreises 2015}

\section{FORSCHUNGSSCHWERPUNKTE}

- Neuronale Regeneration

- Retinale Degeneration

- Reaktive Gliose

- Entwicklung zellbasierter Modelle

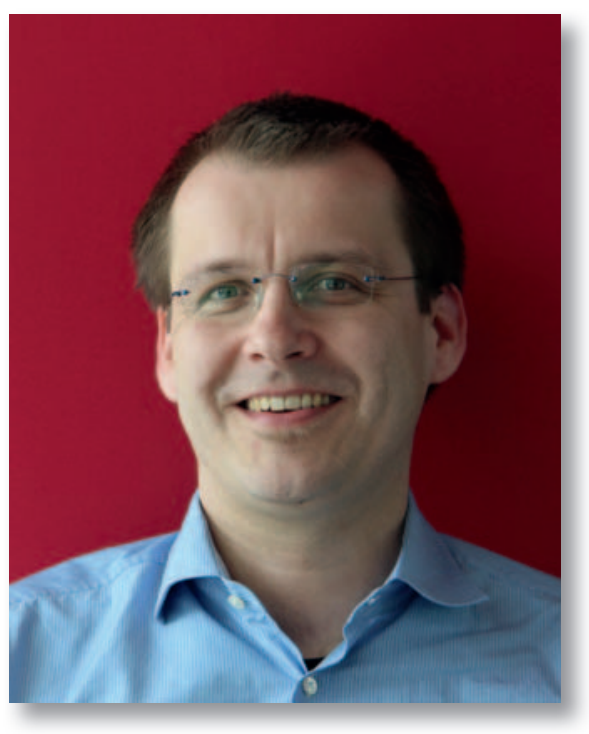

AKADEMISCHER WERDEGANG

- Nachwuchs-Gruppenleiter, Deutsches Zentrum für Neurodegenerative Erkrankungen e.V. (DZNE), Dresden

- Independent Research Associate, Technische Universität Dresden, Center for Regenerative Therapies Dresden CRTD

- Junior Research Fellow, University of Pennsylvania, Philadelphia, PA, USA

- Postdoktorand, University of Washington, Seattle, WA, USA

- Doktorarbeit, Universität Hamburg

- Studium der Humanmedizin, Universität Hamburg
Welche Hypothese lag Ihrer Arbeit «Entwicklung zellbasierter humaner Modelle zur Erforschung retinaler Erkrankungen am Beispiel der Juvenilen Neuronalen Ceroid Lipofuszinose (JNCL)» zugrunde?

Bei der JNCL erleiden die Patienten einen Nervenzell-Verlust im gesamten zentralen Nervensystem; das erste Symptom ist der Sehverlust. Unserer Hypothese nach bietet die JNCL optimale Voraussetzungen für die Entwicklung eines zellbasierten humanen retinalen Erkrankungsmodells in Zellkultur, da sie im Vergleich zu den meisten retinalen Erkrankungen einen sehr frühen Beginn aufweist.

Was war das Hauptergebnis der Arbeit?

Wir haben dreidimensionale humane retinale Gewebe, sogenannte Retinaorganoide, aus humanen induzierten pluripotenten Stammzellen generiert, die aus Hautzellen von gesunden Individuen sowie JNCL-Patienten hergestellt wurden.

Was hat Sie während der Arbeit oder nach Auswertung der Ergebnisse überrascht?

Es ist einfach faszinierend, humane Retina im Labor zu generieren und dies live zu beobachten.

Worin liegt die Relevanz Ihrer Ergebnisse für die praktische, klinische Arbeit eines Ophthalmologen?

Wir betreiben Grundlagenforschung mit dem Ziel, unsere Ergebnisse für die Translationsforschung für regenerative Medizin zu nutzen, um klinisch relevantes Wissen zu generieren und Therapien zu entwickeln.
Für welche Fachgruppen sind Ihre Ergebnisse darüber hinaus interessant?

Dieses neue experimentelle Modell ist zumindest für die klinische Forschung (Ophthalmologie, Neurologie, Physiologie usw.), Stammzell-Forschung und Pharmaindustrie hochrelevant.

Was machen Sie mit dem Preisgeld von 25000 EUR?

Das Projekt steht erst am Anfang und der Forschungspreis unterstützt uns darin, die Ziele zu erreichen, denn die verwendeten Technologien sind hochinnovativ, aber auch noch sehr zeit- und kostenintensiv.

Womit werden Sie sich in naher Zukunft beschäftigen? Unsere Ziele sind die Entwicklung von humanen zellbasierten Retinamodellen und deren erfolgreiche Anwendung für die Erforschung von Erkrankungsmechanismen und Entwicklung von Therapien.

Womit schaffen Sie für sich selbst und für Ihr Umfeld einen Ausgleich zu Ihrem Einsatz in der Forschung?

Meine Familie und der Sport (Mountainbiking) sind ein idealer Ausgleich.

Mit dem EYEnovative Förderpreis sollen innovative Forschungsprojekte aus der Ophthalmologie und benachbarten Wissenschaften unterstützt werden, die das Verständnis hinsichtlich der Ursache, Epidemiologie, Diagnostik und Therapie diverser Netzhaut-Erkrankungen verbessern, um einen direkten Nutzen für Patienten zu generieren. Er wird jährlich von der Novartis Pharma $\mathrm{GmbH}$ ausgeschrieben.

\section{KARGER}

Fax +4976145207 14 information@karger.com www.karger.com
(C) 2015 S. Karger GmbH, Freiburg 2297-0118/15/0012-0095\$39.50/0

Accessible online at: www.karger.com/kop
Dr. Mike O. Karl

${ }^{a}$ Deutsches Zentrum für Neurodegenerative Erkrankungen e.V. (DZNE),

Partnerstandort Dresden, Arnoldstraße 18/18b, 01307 Dresden, Deutschland

${ }^{b}$ Technische Universität Dresden, CRTD - DFG Research Center for Regenerative Therapies Dresden, MOKALAB 2nd FLOOR, R2239, Fetscherstraße 105, 01307 Dresden, Deutschlan mike.karl@dzne.de 